\section{Kurz notiert}

IQWiG bestätigt beträchtlichen Zusatznutzen für Edoxaban $\rightarrow$ Das Institut für Qualität und Wirtschaftlichkeit im Gesundheitswesen (IQWiG) hat Edoxaban (Lixiana ${ }^{\circledR}$ ) einen beträchtlichen Zusatznutzen attestiert. Schlaganfälle, Blutungen und schwere Nebenwirkungen träten unter Edoxaban seltener auf. Der direkte orale Faktor-Xa-Inhibitor ist seit August 2015 in Deutschland verfügbar.

Er wurde im Juni 2015 zur Prävention von Schlaganfällen und systemischen embolischen Ereignissen (SEE) bei erwachsenen Patienten mit nicht-valvulärem Vorhofflimmern (nvVHF) und mindestens einem Risikofaktor zugelassen, sowie zur Behandlung venöser Thromboembolien (VTE; tiefe Beinvenenthrombosen [TVT] und Lungenembolien [LE]) und zur Prävention rezidivierender VTE bei Erwachsenen. Grundlage für die Zulassung waren die Phase-III-Studien ENGAGE AF-TIMI 48 und Hokusai-VTE - die mit 21.105 und 8.292 Patienten größten Zulassungsstudien eines NOAK bei Patienten mit nvVHF bzw. akuter VTE.

Red.

- Nach Informationen von Daiichi-Sankyo

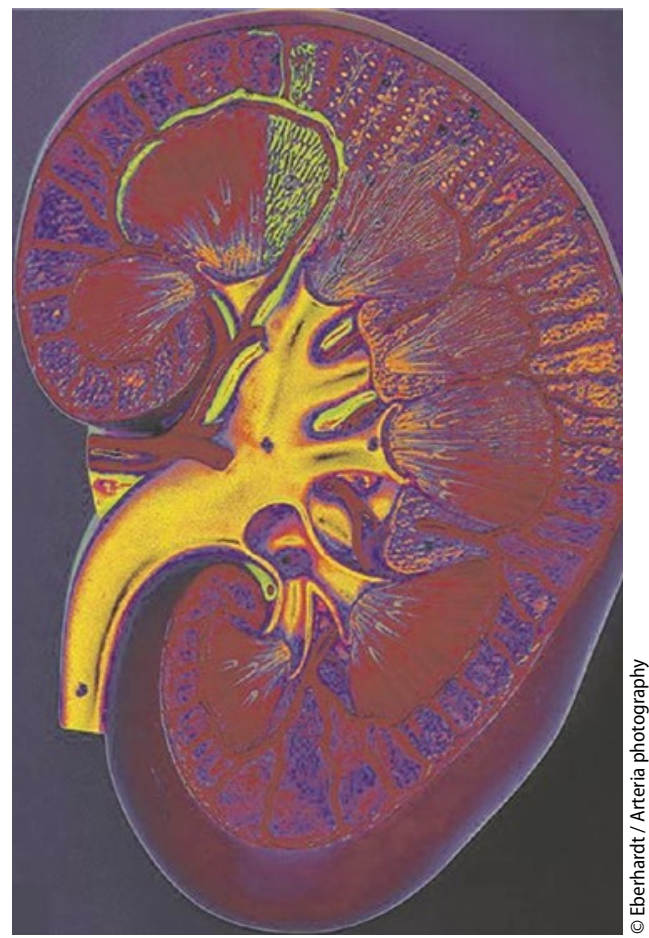

Durch Inhibition der Sodium Dependent Glucose Transporter 2 (SGLT2) wird mehr Glukose mit dem Harn ausgeschieden.

Neue Option für Compliance-schwache Patienten

\title{
Polypille zur KHK-Sekundärprävention
}

Um sich als Patient mit einer koronaren Herzerkrankung (KHK) gegen Komplikationen zu wappnen, muss man $\mathrm{Ni}$ kotin meiden, sich mehr bewegen, auf gesunde Ernährung achten und in der Regel laut Leitlinien mindestens täglich ASS, einen ACE-Hemmer, ein Statin und einen Betablocker einnehmen, so Prof. Uwe Zeymer, Ludwigshafen. Doch die Lebensstilveränderungen fielen furchtbar schwer. Die Therapietreue nimmt mit zunehmender Tablettenanzahl ab und ist bei KHK-Patienten trotz Lebensgefahr schon nach wenigen Jahren erschreckend niedrig.

Ein Weg, die Prognose der oft multimorbiden KHK-Patienten unter Polypharmazie zu verbessern, besteht darin, die lebensverlängernden Wirkstoffe in einer Polypille zu kombinieren. Möglich wird dies nun erstmals mit Sincronium ${ }^{\circledR}$. Die Polypille enthält 100 mg ASS, 20 mg Atorvastatin sowie Ramipril, das mit 2,5 $\mathrm{mg}, 5 \mathrm{mg}$ oder $10 \mathrm{mg}$ eine individuelle Dosierung ermöglicht.

\section{Deutliche Steigerung der Adhärenz}

Nach den Worten von Prof. Peter Bramlage, Institut für Pharmakologie und präventive Medizin in Mahlow, wurde in einer Studie nachgewiesen, dass die Polypille die Adhärenz nach einem Jahr im Vergleich zu einer Therapie mit Einzelsubstanzen absolut um $10 \%$ verbessert. „10\% mehr Adhärenz reduzieren die Rate kardiovaskulärer Komplikationen um 6,7\%", so Bramlage. Eine gute Therapietreue zur Polypille von über $80 \%$ senkt die Sterblichkeit gegenüber keiner Therapie gar um $71 \%$ und gegenüber Monotherapien mit ASS oder Statinen um 25-30\%.

Dr. Dirk Einecke

- Fachpressesymposium \& Meet-the-Expert "Klinischer Nutzen der Polypille - wie effektive Sekundärprävention wirkt", DGKHerbsttagung; Berlin, Oktober 2015 (Veranstalter: Hexal)

\section{SGLT2-Hemmer bei Diabetes mellitus Typ 2}

\section{Neuer Anlauf für eine Nutzenbewertung}

_ Dem SGLT2-Hemmer Dapagliflozin war 2013 vom Gemeinsamen Bundesausschuss (G-BA) aus formellen Gründen kein Zusatznutzen bescheinigt worden, weil die Zulasssungsstudie Mängel aufgewiesen habe.

Jetzt hat AstraZeneca die DapaZuStudie gestartet. Die neue Studie berücksichtigt die formalen Kritikpunkte des G-BA. Dazu werden bis Ende des Jahres 930 Typ-2-Diabetiker gesucht. Das Unternehmen appelliert an niedergelassene Ärzte, geeignete Patienten an die Studienzentren zu vermitteln.

Ziel der Untersuchung ist es, Wirksamkeit und Verträglichkeit von Dapagliflozin mit der von Glimepirid (jeweils in Kombination mit Metformin) zu vergleichen. Der Sulfonylharnstoff ist die vom G-BA gewünschte Vergleichssubs$\operatorname{tanz}$. Die Patienten werden dabei rando- misiert in drei gleich große Gruppen aufgeteilt und erhalten über 52 Wochen verblindet je drei Tabletten plus Metformin (mindestens $1.500 \mathrm{mg}$ ): Dapagliflozin 10 mg (plus zweimal Placebo), Dapagliflozin $10 \mathrm{mg}$ plus Saxagliptin $5 \mathrm{mg}$ plus einmal Placebo, Glimepirid (auftitiert 1, 2 oder 4 mg) plus zweimal Placebo.

Primärer Endpunkt ist die Veränderung des $\mathrm{HbA}_{1 \mathrm{c}}$-Wertes, wie Prof. Dirk Müller-Wieland, Hamburg, berichtete. „Sekundäre Endpunkte sind der Anteil der Patienten mit mindestens einer Hypoglykämie, Veränderungen bei Körpergewicht und Nüchternplasmaglukose sowie die Zeit bis zur Insulingabe." -

Wolfgang Geissel

- Pressekonferenz „DapaZu-Studie in Deutschland - für einen Zusatznutzen und neue Perspektiven von Dapagliflozin in der Therapie des Typ 2 Diabetes; Frankfurt/M., Oktober 2015 (Veranstalter: AstraZeneca) 